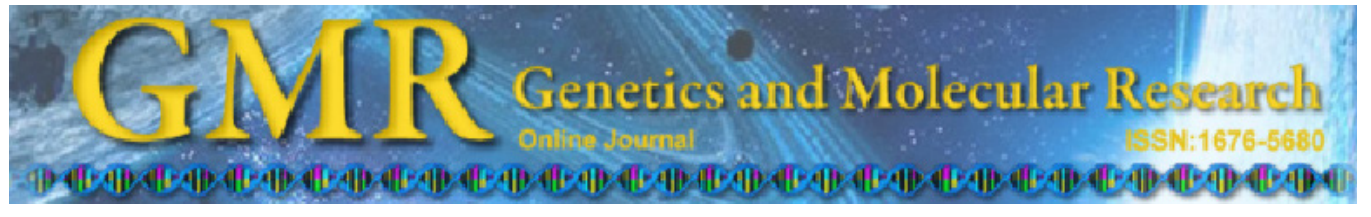

\title{
Enhancement of recombinant adeno-associated virus mediated transgene expression by targeted echo-contrast agent
}

S.L. Yang ${ }^{1,3}$, Y.M. Mu ${ }^{1}$, K.Q. Tang ${ }^{3}$, X.K. Jiang ${ }^{1}$, W.K. Bai ${ }^{2}$, E. Shen ${ }^{2}$ and B. Hu

${ }^{1}$ Department of Echocardiography, First Teaching Hospital,

Xinjiang Medical University, Urumqi, China;

${ }^{2}$ Department of Ultrasound in Medicine,

Shanghai Institute of Ultrasound in Medicine, Affiliated 6th People's

Hospital, Shanghai Jiao Tong University, Shanghai, China

${ }^{3}$ Department of Ultrasound in Medicine,

Shanghai Institute of Ultrasound in Medicine,

Affiliated 6th People's South Hospital, Shanghai Jiao Tong University,

Shanghai, China

Corresponding authors: Y.M. Mu / B. Hu

E-mail: yu-mingmu@hotmail.com / binghu_stephen@163.com

Genet. Mol. Res. 12 (2): 1318-1326 (2013)

Received July 16, 2012

Accepted January 22, 2013

Published April 25, 2013

DOI http://dx.doi.org/10.4238/2013.April.25.3

\begin{abstract}
Ultrasound-targeted microbubble destruction (UTMD) has been recently developed for destroying bubbles carrying drugs or genes, thereby permitting local release of these target molecules. We investigated whether SonoVue ${ }^{\circledR}$, a new contrast agent that contains phospholipid-stabilized microbubbles filled with sulfur hexafluoride vapor, is effective at delivering a recombinant adeno-associated viral (rAAV) vector to the rat heart by UTMD. Serotype-2 (rAAV2) marked with green fluorescent protein (GFP) as a reporter gene was attached to the surface of sulfur hexafluoride-filled microbubbles. Microbubbles were infused into the tail vein of rats with or without simultaneous echocardiography. Additional controls included ultrasound microbubbles that did not contain virus, virus alone, and virus plus ultrasound. One group underwent echocardiographic destruction of microbubbles followed by rAAV2-GFP infusion. Rats were killed after 4 weeks and examined for GFP expression. Green fluorescence was detected in all groups that received the rAAV2-GFP vector, indicating expression of the rAAV2
\end{abstract}


transgene; however, GFP expression in the UTMD group was significantly higher than that in control groups. We conclude that ultrasoundmediated destruction mediated by SonoVue is a promising method for delivery of rAAV2 to the heart in vivo.

Key words: Ultrasound; Recombinant adeno-associated virus; Microbubble; Green fluorescent protein

\section{INTRODUCTION}

Gene transfer has been considered a promising therapeutic tool in several clinical trials for about two decades. However, successful gene therapy remains an elusive and challenging goal. Importantly, several clinical trials of cardiac gene therapy have mostly lacked relevant clinical and physiological efficacy (Bekeredjian and Shohet, 2004; Müller et al., 2007). The success of cardiac gene therapy may be affected by suitable candidate genes, vector design, vector type and mode of vector delivery.

The two most commonly used vectors in cardiac gene therapy are naked DNA and adeno-associated virus (AAV) vectors. In comparing these two vectors, AAV has the advantage of being suitable for long-term gene transfer for at least 1 year in the myocardium (Chu et al., 2003; Vassalli et al., 2003). During the past 20 years, recombinant adeno-associated virus serotype 2 (rAAV2) has been successfully used in $70 \%$ of experimental therapeutic approaches (Edelstein et al., 2004), such as protection from ischemia/reperfusion injury in a rat model (Melo et al., 2002) or inotropic therapy in cardiomyopathic hamsters (Kawada et al., 2002), and has revealed beneficial effects on cardiac function in a murine model of myocardial ischemia (Su et al., 2004). To achieve much more highly selective transduction of myocardial tissue, rAAV2 vectors were often administered by intramyocardial injection (Rutanen et al., 2004) or selective perfusion of coronary arteries (Raake et al., 2008). These techniques are invasive and have inherent risk. Therefore, the development of noninvasive but highly organspecific systemic gene transfer approaches has been a major focus.

Recently, ultrasound-targeted microbubble destruction (UTMD) has evolved into a new tool for organ-specific delivery of genes and drugs (Hiyama et al., 2007; Aoi et al., 2008). It is based on gas-filled microbubbles that are used as ultrasound contrast agents. Microbubbles may oscillate in an ultrasound field when submitted to ultrasound at their resonance frequency. Low and intermediate acoustic pressure results in linear and nonlinear oscillations of microbubbles, respectively, whereas high-pressure ultrasound (mechanical index $>1.0$ ) causes forced expansion and compression of microbubbles, leading to bubble destruction (Dijkmans et al., 2004). Bioactive substances loaded on the microbubble shells can be released by UTMD with high local concentrations in the target organ ( $\mathrm{Li}$ et al., 2008; Wang et al., 2008; Delalande et al., 2010). In addition, UTMD benefits from the secondary effects of microbubble destruction, transiently increasing capillary permeability and producing transient nanopores in cell membranes by high-velocity fluid microjets (Bekeredjian et al., 2007; Suzuki et al., 2008; Geis et al., 2009), thus augmenting transduction. As expected, Bekeredjian et al. (2005) demonstrated that rats receiving ultrasound and luciferase-loaded bubbles showed a 6-fold higher cardiac luciferase uptake compared with control groups that did not include bubbles. Müller et al. (2008) showed that in contrast to low cardiac expression after systemic transfer of the AAV6 or -9 vector solution without microbubbles, ultrasound-targeted destruction of microbubbles 
significantly increased cardiac reporter activities between 6- and 20-fold.

In this study, 6 different groups were designed to evaluate whether UTMD could augment rAAV2-mediated gene transfer to the heart. rAAV2 vector is a lower transduction vector for heart compared with AAV-6 and AAV-9. Null rAAV2 vector was used for this study to prevent a candidate gene target effect and to confirm that the target effect only resulted from UTMD treatment. We tested if systemic administration of rAAV2-GFP (green fluorescent protein) vectors could lead to more efficient gene expression in rat heart when using UTMD. Furthermore, we investigated whether UTMD-enhanced rAAV2 delivery to the myocardium increases organ specificity.

\section{MATERIAL AND METHODS}

\section{Construction of rAAV2-GFP vector}

A single-stranded, 7015-bp rAAV2-GFP vector (Vector Gene Technology Company Limited, Beijing, China) was constructed as described by Wu et al. (2000). Briefly, viral replicable genes, rep and cap, were replaced with a human cytomegalovirus promoter and enhancer, a multiple-cloning site and a neomycin-resistant gene, as well as a polyadenylation signal, retaining the two inverted terminal repeats intact. The GFP reporter gene was inserted into the site between $S a l I$ and $B g l I I$ in the multiple-cloning site. After screening by neomycin with the help of mutated herpes simplex virus (HSV)-1, large-scale rAAV2 were produced and purified by affinity chromatography and ultracentrifugation in $\mathrm{CsCl}$ gradients. Viral titer was determined by dot blot hybridization. The helper virus HSV was heat-inactivated for $30 \mathrm{~min}$ at $56^{\circ} \mathrm{C}$. Contaminating HSV was determined to be $<1$ in $10^{10}$ viral genomes (vg).

\section{Attachment of rAAV2-GFP to microbubbles}

AAV2-GFP-loaded microbubbles were produced as follows. SonoVue (Bracco SpA, Milan, Italy) is a kind of ultrasound microbubble contrast agent that contains $59 \mathrm{mg}$ sulfur hexafluoride gas and $25 \mathrm{mg}$ freeze-dried white powder. After adding $5 \mathrm{~mL} 0.9 \% \mathrm{NaCl}$ into the vial with SonoVue and shaking for several seconds, phospholipid-stabilized microbubble suspensions were generated (Xu et al., 2006). A 1-mL solution of rAAV2-GFP $\left(1.5 \times 10^{11} \mathrm{vg} /\right.$ $\mathrm{mL}$ ) was incubated with $1 \mathrm{~mL}$ microbubble suspension for $2 \mathrm{~h}$ at $4^{\circ} \mathrm{C}$. The concentration and mean diameter of microbubbles with attached rAAV2-GFP were $1.2 \times 10^{9}$ bubbles $/ \mathrm{mL}$ and 2.5 $\mu \mathrm{m}$, respectively. The microbubble suspension contained $1.5 \times 10^{11} \mathrm{vg}$ rAAV2-GFP transgene units per milliliter.

\section{In vivo gene transfer}

All procedures were approved by the Local Animal Care Committee. A total of 60 Sprague-Dawley rats (250-350 g) were used for our experiments. They were randomly divided into 6 groups $(\mathrm{N}=10)$ to determine the effectiveness of in vivo delivery of rAAV2-GFP into the myocardium using UTMD: group 1, echocardiographic destruction of microbubbles without rAAV2-GFP; group 2, infusion of rAAV2-GFP (no microbubbles) without echocardiography; group 3, echocardiography during infusion of rAAV2-GFP (no microbubbles); group 
4, echocardiographic destruction of microbubbles followed by rAAV2-GFP infusion (when microbubbles were seen in the myocardium, high mechanical index $(\mathrm{MI}=1.6)$ ultrasound was applied to destroy the bubbles, and after no bubbles were seen in the myocardium and heart, rAAV2-GFP was infused); group 5, microbubbles containing rAAV2-GFP without echocardiography; group 6, echocardiographic destruction of microbubbles containing rAAV2-GFP. We administered infusions $(1.2 \mathrm{~mL})$ over $20 \mathrm{~min}$ because of the risk of volume overload.

Rats were anesthetized with an intraperitoneal injection of $50 \mathrm{mg} / \mathrm{kg}$ sodium pentobarbital. After shaving and depilating the left chest wall, the transducer was placed on the thorax in a longitudinal axis view, with 1-2 cm of acoustic coupling gel standoff between the transducer and chest wall. The microbubble suspension ( $1 \mathrm{~mL}$ SonoVue) alone, microbubble and virus $(1 \mathrm{~mL})$ suspension, and pure virus $(1 \mathrm{~mL})$ suspension were separately infused into the tail vein at a constant rate of $3 \mathrm{~mL} / \mathrm{h}$ for $20 \mathrm{~min}$. During the 20 -min infusion, the bubbles were mixed in the same incubated viral suspension and then bubbles were infused into the syringe. Microbubble destruction was achieved using a GE VIVID7 Dimension (General Electric Co., Horten, Norway) echocardiographic color system, equipped with a 2.0-3.5-MHz electronic phased array transducer (M3S) and a 10-14-MHz electronic linear array transducer (i13L). The latter was used to locate the heart with low-energy $(\mathrm{MI}=0.14)$ imaging at $11-13$ frames per second during infusion of microbubbles or control into the tail vein. A low dynamic range and pulse-repetition frequency of $50 \mathrm{~Hz}$ was selected to achieve definite visible myocardial opacification on the basis of pilot experiments. After the microbubbles became visible in the myocardium, the M3S transducer was operated in a second harmonic mode (transmit 1.3 $\mathrm{MHz} /$ receive $2.6 \mathrm{MHz}$ ) at an MI of 1.6 (corresponding to a peak negative pressure of about $1.8 \mathrm{MPa}$ at a depth of $2 \mathrm{~cm}$ ). Sonography was electrocardiogram triggered, at $80 \mathrm{~ms}$ after the peak of the R-wave, to deliver a burst of four frames of ultrasound (with 4 cycles per frame) every 4 heartbeats.

\section{Fluorescence measurement}

Rats were euthanized 4 weeks after rAAV2-GFP transfection, at which time peak levels of transgene expression in heart occurred. The hearts and brain and liver tissues were harvested and immediately frozen in liquid nitrogen for fluorescence measurement. Fluorescence signals of GFP-positive fibers were examined using fluorescence microscopy (Leica CTR6000, Wetzlar, Germany) with an excitation wavelength of $488 \mathrm{~nm}$ (Argon laser, 5\% intensity). Fluorescence images were captured at an emission wavelength of 505-530 nm to detect GFP. Non-specific fluorescence was visualized at $>560 \mathrm{~nm}$. Settings for gain and offset were optimized using tissue from rAAV2-GFP-transgenic and control rats as positive and negative controls. Readout was performed by the principal microscopist using 15 sections randomly selected from 200 slides by a person not involved in the study and not knowledgeable of the group assignment of rats (Storek et al., 2006). Slides included sections from all parts of the heart and were in approximately equal proportion from rat infusion with rAAV2-GFP or SonoVue only. Integrated optical density (IOD) of fluorescence images was measured using the Image-Pro Plus 6.0 software. Fluorescence intensity was expressed in arbitrary units. IOD was calculated by applying a digital mask over regions where GFP was expressed. Fluorescence was corrected for background by placing mask "objects" in the field outside the fluorescence area and IOD of these objects subtracted from the IOD of the region of interest manager 
(Juffermans et al., 2008). Threshold and segmentation tools were used to extract objects of interest and distinguish them from other objects or from the background image.

\section{Statistical analysis}

All data are reported as means \pm standard deviation (SD) and analyzed by the SPSS13.0 software. Differences in IOD of fluorescence between experimental groups were compared by ANOVA. The Student $t$-test was used to assess the statistical difference between multiple comparisons. $\mathrm{P}<0.05$ was considered to be significant.

\section{RESULTS}

The influence of UTMD on transduction efficiency of rAAV2 serotype was investigated by GFP reporter gene expression. Green fluorescence was detected in groups 2-5 because they all received the rAAV2-GFP vector (Figure 1). However, GFP expression was significantly higher in group 3 compared with group $2(\mathrm{P}=0.001$; Figure 2$)$, indicating that ultrasonic treatment improved gene delivery although without microbubbles. There was no significant difference between group 5 and group $2(\mathrm{P}=0.939)$, suggesting that ultrasonic is a significant factor in achieving rAAV2-GFP transduction although rAAV2-GFP was infused with microbubbles in group 5. As expected, we found that GFP expression was approximately 6.5- to 10-fold higher in the UTMD group (group 6) compared to control groups (groups 2, 3,4 , and 5) receiving rAAV2-GFP but without microbubbles or ultrasound. The group that underwent microbubble destruction followed by an infusion of rAAV-GFP (group 4) showed a 1.5-fold increase in GFP expression compared with other controls (groups 2, 3, and 5), suggesting that disruption of the endothelial barrier is an important factor in viral transduction.
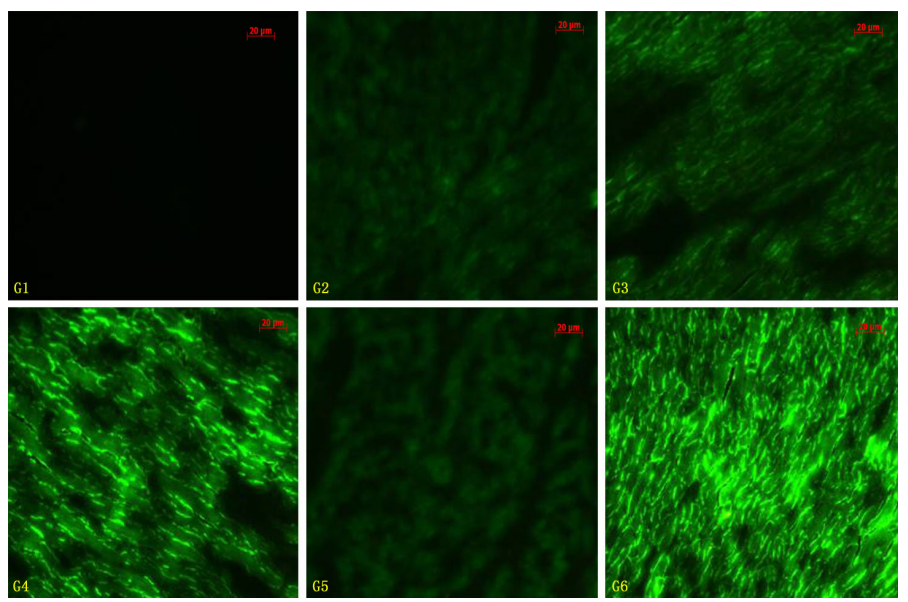

Figure 1. Green fluorescent protein (GFP) expression in frozen section of rat myocardium (400X). G1 = Echocardiographic destruction of microbubbles without recombinant adeno-associated virus serotype-2 (rAAV2GFP). G2 = Infusion of rAAV2-GFP (no microbubbles) without echocardiography. G3 = Echocardiography during infusion of rAAV2-GFP (no microbubbles). G4 = Echocardiographic destruction of microbubbles followed by rAAV2-GFP infusion. G5 = Infusion of microbubbles containing rAAV2-GFP without echocardiography. G6 = Echocardiographic destruction of microbubbles containing rAAV2-GFP. 


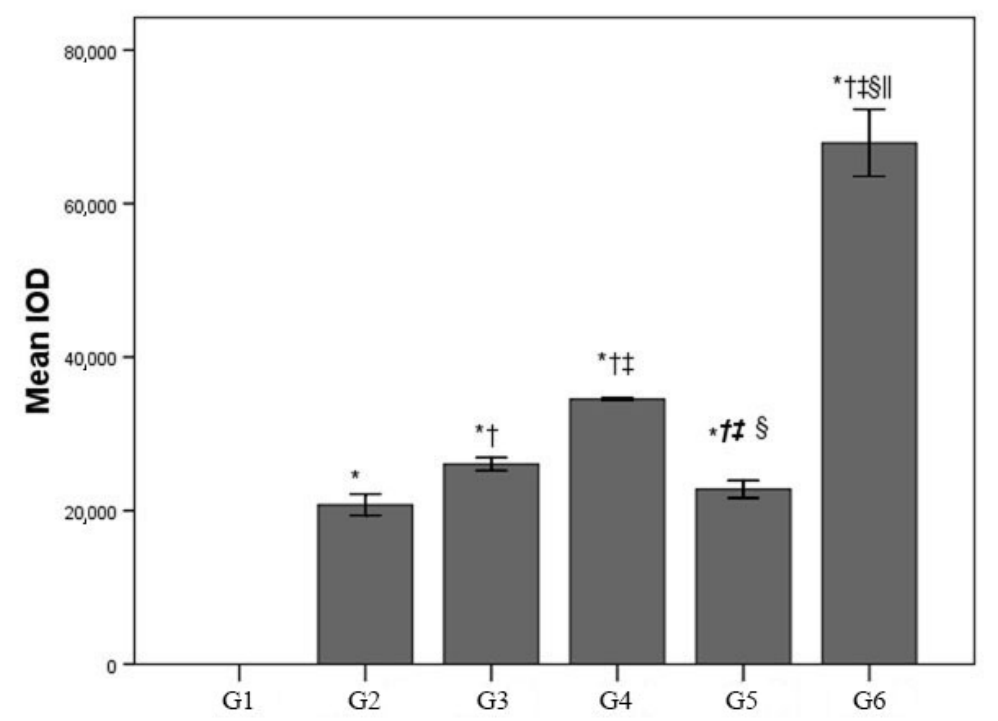

Figure 2. Statistical analysis of green fluorescent protein expression in frozen section of rat myocardium. *Significant difference compared with G1 $(\mathrm{P}=0.0001)$; † significant difference compared with $\mathrm{G} 2(\mathrm{P}=0.0001)$; tsignificant difference compared with G3 ( $\mathrm{P}=0.0001)$; §significant difference compared with G4 $(\mathrm{P}=0.0001)$; ||significant difference compared with G5 $(\mathrm{P}=0.0001)$; tno significant difference compared with G2 $(\mathrm{P}=0.939)$; tsignificant difference compared with G3 $(\mathrm{P}=0.038)$. IOD = integrated optical density. For group definitions $(\mathrm{G} 1$ G6), see legend to Figure 1.

In addition, fluorescence analysis of various organs (heart, liver and brain) of animals euthanized after 4 weeks indicated that GFP was expressed in liver tissue and myocardium but not in brain tissue in groups 2-6 (Figure 3). However, GFP expression in the UTMD group was significantly higher in myocardium than in liver, indicating target organ-specific rAAV2-GFP vector delivery using UTMD.

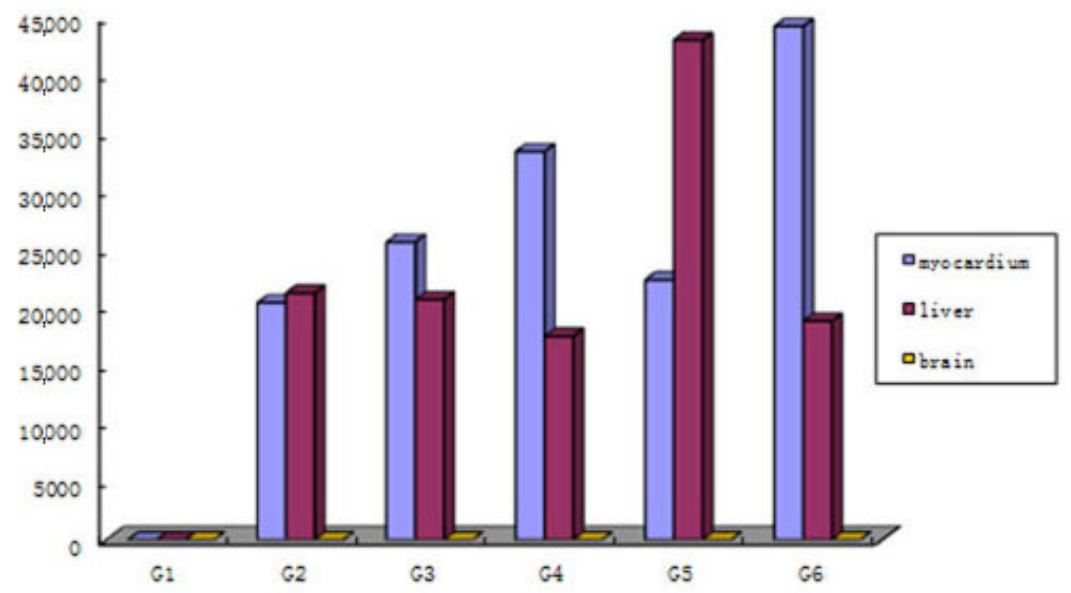

Figure 3. Green fluorescent protein expression in frozen section of rat myocardium, liver and brain. For group definitions (G1-G6), see legend to Figure 1. 


\section{DISCUSSION}

Previously, several investigators have successfully transfected the heart with lipopolymers (Lee et al., 2003), gelatin complexes (Tokunaga et al., 2004) and lipoprotein (Harriss and Atkinson, 2011). Intravenous administration of DNA-carrying liposomes leads to the transfection of many organs, particularly the lung (Boussif et al., 2001). In this study, we delivered rAAV2-GFP to the heart by UTMD. The findings demonstrated that high levels of myocardial GFP expression were achieved after delivery of rAAV2-GFP transgene using UTMD. GFP expression in the UTMD group was significantly higher than that in other control groups $(\mathrm{P}=$ 0.001 ), approximately 6.5 - to 10 -fold increase. There was no significant difference between groups 2 and 5, both of which did not undergo echocardiography. The present study confirmed the usefulness of this technique for the delivery of a common gene-therapy vector that is capable of high-level expression of a transgene in the heart.

Several researchers have shown that ultrasound and microbubbles may have important applications in gene therapy (Unger et al., 2001; Miura et al., 2002). The present study demonstrated high levels of GFP gene expression using systemic intravenous infusion of rAAV2-GFPmicrobubbles during echocardiography. The advantages of this much less invasive approach in cardiac gene therapy are apparent. UTMD-directed gene delivery to specific organs would be a substantial improvement in the therapeutic ratio for a broad range of genetic manipulations.

rAAV2 vectors have rapidly gained popularity in gene therapy applications. Exchanging capsids between various AAV serotypes is effective for conferring specific cellular tropism and intensity, as well as onset of gene expression (Coura and Nardi, 2007; Surace and Auricchio, 2008). Virus-mediated gene delivery has resulted in higher levels of cardiac transduction (about 30- to 360-fold) compared to plasmid approaches after direct intramyocardial injections in rabbits (Wright et al., 2001). Widespread use of such vectors is compromised by the lack of specificity to target cells via systemic administration (Taylor et al., 2007). We attempted to combine the advantages of rAAV2, ultrasound and microbubbles (SonoVue) to enhance GFP transgene to the heart via systemic administration. Significant expression of GFP was detected in the UTMD group. Treatment groups 2-5 with rAAV2-GFP infusion showed a lower level of expression than the group 6. The increased transduction efficiency in the UTMD group can be explained by enhanced local extravasation of the virus after destruction of AAVloaded microbubbles in the cardiac capillaries and increased capillary permeability resulting from high-velocity fluid microjets (Bazan-Peregrino et al., 2012).

Under electrostatic interaction, materials may be non-covalently bound to the surface of microbubbles (Unger et al., 2001). We modified the rAAV2-GFP transgene by electrostatic interaction using SonoVue. This enhanced rAAV2-GFP delivery, making it convenient for applying it in UTMD. Transthoracic ultrasound applying the M3S transducer can be focused on the heart so that the destruction of intravenously injected gene-bearing microbubbles by high-energy ultrasound delivers genes relatively selectively to the myocardium. As expected, our results indicated that GFP expression in the UTMD group was significantly higher in myocardium than in liver and brain, indicating target organ-specific rAAV2-GFP vector delivery using UTMD, which was in accordance with the results of Müller et al. (2007), who also found high luciferase activities in left ventricles and atria but close to background levels in other organs. Our data provide not only the means of achieving stable ultrasound/microbubblemediated gene delivery but also a solution to the critical issue of specificity after systemic administration of rAAV2-GFP. 
The combination of ultrasound/microbubbles and rAAV2-GFP is efficient. Microbubble accumulation and destruction at the target site can be monitored using ultrasound imaging to assess vector delivery in real-time. The i13L transducer was used to monitor microbubble accumulation in the myocardium. Systemic infusion of targeted vectors may play an important role in the future. Varying the ultrasound parameter will probably affect the efficiency of UTMD-targeted gene delivery. Further preclinical studies in larger animals are required.

\section{CONCLUSIONS}

We demonstrated that UTMD after systemic administration can enhance targeted gene transfer to the heart. It is an efficient, specific tissue-targeting, non-invasive approach to deliver genes to target organs by UTMD after systemic administration.

\section{ACKNOWLEDGMENTS}

Research supported in part by the Animal Care Center of the Affiliated Hospital of Xinjiang Medical University and the National Natural Science Foundation of China (grant \#30660181). SonoVue was purchased from Bracco SpA, Milan, Italy.

\section{REFERENCES}

Aoi A, Watanabe Y, Mori S, Takahashi M, et al. (2008). Herpes simplex virus thymidine kinase-mediated suicide gene therapy using nano/microbubbles and ultrasound. Ultrasound Med. Biol. 34: 425-434.

Bazan-Peregrino M, Arvanitis CD, Rifai B, Seymour LW, et al. (2012). Ultrasound-induced cavitation enhances the delivery and therapeutic efficacy of an oncolytic virus in an in vitro model. J. Control Release 157: 235-242.

Bekeredjian R and Shohet RV (2004). Cardiovascular gene therapy: angiogenesis and beyond. Am. J. Med. Sci. 327 : 139-148.

Bekeredjian R, Chen S, Grayburn PA and Shohet RV (2005). Augmentation of cardiac protein delivery using ultrasound targeted microbubble destruction. Ultrasound Med. Biol. 31: 687-691.

Bekeredjian R, Kuecherer HF, Kroll RD, Katus HA, et al. (2007). Ultrasound-targeted microbubble destruction augments protein delivery into testes. Urology 69: 386-389.

Boussif O, Gaucheron J, Boulanger C, Santaella C, et al. (2001). Enhanced in vitro and in vivo cationic lipid-mediated gene delivery with a fluorinated glycerophosphoethanolamine helper lipid. J. Gene Med. 3: 109-114.

Chu D, Sullivan CC, Weitzman MD, Du L, et al. (2003). Direct comparison of efficiency and stability of gene transfer into the mammalian heart using adeno-associated virus versus adenovirus vectors. J. Thorac. Cardiovasc. Surg. 126: 671-679.

Coura RS and Nardi NB (2007). The state of the art of adeno-associated virus-based vectors in gene therapy. Virol J. 4: 99.

Delalande A, Bureau MF, Midoux P, Bouakaz A, et al. (2010). Ultrasound-assisted microbubbles gene transfer in tendons for gene therapy. Ultrasonics 50: 269-272.

Dijkmans PA, Juffermans LJ, Musters RJ, van Wamel A, et al. (2004). Microbubbles and ultrasound: from diagnosis to therapy. Eur. J. Echocardiogr. 5: 245-256.

Edelstein ML, Abedi MR, Wixon J and Edelstein RM (2004). Gene therapy clinical trials worldwide 1989-2004-an overview. J. Gene Med. 6: 597-602.

Geis NA, Mayer CR, Kroll RD, Hardt SE, et al. (2009). Spatial distribution of ultrasound targeted microbubble destruction increases cardiac transgene expression but not capillary permeability. Ultrasound Med. Biol. 35: 1119-1126.

Harriss DJ and Atkinson G (2011). Update - Ethical standards in sport and exercise science research. Int. J. Sports Med. 32: 819-821.

Hiyama A, Mochida J, Iwashina T, Omi H, et al. (2007). Synergistic effect of low-intensity pulsed ultrasound on growth factor stimulation of nucleus pulposus cells. J. Orthop. Res. 25: 1574-1581.

Juffermans LJ, Kamp O, Dijkmans PA, Visser CA, et al. (2008). Low-intensity ultrasound-exposed microbubbles provoke local hyperpolarization of the cell membrane via activation of BK(Ca) channels. Ultrasound Med. Biol. 34: 502-508. 
Kawada T, Nakazawa M, Nakauchi S, Yamazaki K, et al. (2002). Rescue of hereditary form of dilated cardiomyopathy by rAAV-mediated somatic gene therapy: amelioration of morphological findings, sarcolemmal permeability, cardiac performances, and the prognosis of TO-2 hamsters. Proc. Natl. Acad. Sci. U. S. A. 99: 901-906.

Lee M, Rentz J, Bikram M, Han S, et al. (2003). Hypoxia-inducible VEGF gene delivery to ischemic myocardium using water-soluble lipopolymer. Gene Ther. 10: 1535-1542.

Li X, Wang Z, Ran H, Li X, et al. (2008). Experimental research on therapeutic angiogenesis induced by hepatocyte growth factor directed by ultrasound-targeted microbubble destruction in rats. J. Ultrasound Med. 27: 453-460.

Melo LG, Agrawal R, Zhang L, Rezvani M, et al. (2002). Gene therapy strategy for long-term myocardial protection using adeno-associated virus-mediated delivery of heme oxygenase gene. Circulation 105: 602-607.

Miura S, Tachibana K, Okamoto T and Saku K (2002). In vitro transfer of antisense oligodeoxynucleotides into coronary endothelial cells by ultrasound. Biochem. Biophys. Res. Commun. 298: 587-590.

Müller OJ, Katus HA and Bekeredjian R (2007). Targeting the heart with gene therapy-optimized gene delivery methods. Cardiovasc. Res. 73: 453-462.

Müller OJ, Schinkel S, Kleinschmidt JA, Katus HA, et al. (2008). Augmentation of AAV-mediated cardiac gene transfer after systemic administration in adult rats. Gene Ther. 15: 1558-1565.

Raake PW, Hinkel R, Muller S, Delker S, et al. (2008). Cardio-specific long-term gene expression in a porcine model after selective pressure-regulated retroinfusion of adeno-associated viral (AAV) vectors. Gene Ther. 15: 12-17.

Rutanen J, Rissanen TT, Markkanen JE, Gruchala M, et al. (2004). Adenoviral catheter-mediated intramyocardial gene transfer using the mature form of vascular endothelial growth factor-D induces transmural angiogenesis in porcine heart. Circulation 109: 1029-1035.

Storek B, Harder NM, Banck MS, Wang C, et al. (2006). Intrathecal long-term gene expression by self-complementary adeno-associated virus type 1 suitable for chronic pain studies in rats. Mol. Pain 2: 4.

Su H, Joho S, Huang Y, Barcena A, et al. (2004). Adeno-associated viral vector delivers cardiac-specific and hypoxiainducible VEGF expression in ischemic mouse hearts. Proc. Natl. Acad. Sci. U. S. A. 101: 16280-16285.

Surace EM and Auricchio A (2008). Versatility of AAV vectors for retinal gene transfer. Vision Res. 48: 353-359.

Suzuki R, Takizawa T, Negishi Y, Utoguchi N, et al. (2008). Effective gene delivery with novel liposomal bubbles and ultrasonic destruction technology. Int. J. Pharm. 354: 49-55.

Taylor SL, Rahim AA, Bush NL, Bamber JC, et al. (2007). Targeted retroviral gene delivery using ultrasound. J. Gene Med. 9: 77-87.

Tokunaga N, Nagaya N, Shirai M, Tanaka E, et al. (2004). Adrenomedullin gene transfer induces therapeutic angiogenesis in a rabbit model of chronic hind limb ischemia: benefits of a novel nonviral vector, gelatin. Circulation 109: 526531.

Unger EC, Hersh E, Vannan M, Matsunaga TO, et al. (2001). Local drug and gene delivery through microbubbles. Prog. Cardiovasc. Dis. 44: 45-54.

Vassalli G, Bueler H, Dudler J, von Segesser LK, et al. (2003). Adeno-associated virus (AAV) vectors achieve prolonged transgene expression in mouse myocardium and arteries in vivo: a comparative study with adenovirus vectors. Int. J. Cardiol. 90: 229-238.

Wang JF, Wang JB, Chen H, Zhang CM, et al. (2008). Ultrasound-mediated microbubble destruction enhances gene transfection in pancreatic cancer cells. Adv. Ther. 25: 412-421.

Wright MJ, Wightman LM, Lilley C, de Alwis M, et al. (2001). In vivo myocardial gene transfer: optimization, evaluation and direct comparison of gene transfer vectors. Basic Res. Cardiol. 96: 227-236.

Wu XB, Dong XY and Wu ZJ (2000). A novel technique for adeno-associated virus purification. Chin. Sci. Bull. 45: 2071-2075.

Xu HX, Liu GJ, Lu MD, Xie XY, et al. (2006). Characterization of small focal liver lesions using real-time contrastenhanced sonography: diagnostic performance analysis in 200 patients. J. Ultrasound Med. 25: 349-361. 